\title{
Statistical analysis of image quality measures for face liveness detection
}

\begin{abstract}
Face recognition is essential for a wide range of technologies that requires person identification. Due to the presence of spoof face attacks, an additional layer of security is needed to protect the system, which can be provided by liveness detection. In this paper we develop a technique for discriminating live from fake images. Our approach is based upon the hypothesis that spoofing scheme leave statistical indication or structure in images which can be utilized for detection by assistance of image quality features. To achieve this, image quality measures (IQMs) statistical evaluation has been implemented using the analysis of variance (ANOVA) technique. A feature set of measures with highest discrimination power to distinguish between real and fake images was obtained. This ensures the simplicity of detection system and improves its computational efficiency.
\end{abstract}

Keyword : Analysis of variance; Image quality measures; Face liveness detection 